EPJ Web of Conferences 86,00066 (2015)

DOI: $10.1051 /$ epjconf/ 20158600066

(C) Owned by the authors, published by EDP Sciences, 2015

\title{
Synthesis of superheavy nuclei: Obstacles and opportunities
}

\author{
V.I. Zagrebaev ${ }^{1, a}$, A.V. Karpov ${ }^{1, b}$, and Walter Greiner $^{2, c}$ \\ ${ }^{1}$ Flerov Laboratory of Nuclear Reactions, JINR, Dubna, Moscow Region, Russia \\ ${ }^{2}$ Frankfurt Institute for Advanced Studies, J.W. Goethe-Universität, Frankfurt, Germany
}

\begin{abstract}
There are only 3 methods for the production of heavy and superheavy (SH) nuclei, namely, fusion reactions, a sequence of neutron capture and beta(-) decay and multinucleon transfer reactions. Low values of the fusion cross sections and very short half-lives of nuclei with $\mathrm{Z}>120$ put obstacles in synthesis of new elements. At the same time, an important area of SH isotopes located between those produced in the cold and hot fusion reactions remains unstudied yet. This gap could be filled in fusion reactions of ${ }^{48} \mathrm{Ca}$ with available lighter isotopes of $\mathrm{Pu}, \mathrm{Am}$, and $\mathrm{Cm}$. New neutron-enriched isotopes of $\mathrm{SH}$ elements may be produced with the use of a ${ }^{48} \mathrm{Ca}$ beam if a ${ }^{250} \mathrm{Cm}$ target would be prepared. In this case we get a real chance to reach the island of stability owing to a possible beta(+) decay of ${ }^{291} 114$ and ${ }^{287} 112$ nuclei formed in this reaction with a cross section of about $0.8 \mathrm{pb}$. A macroscopic amount of the long-living SH nuclei located at the island of stability may be produced by using the pulsed nuclear reactors of the next generation only if the neutron fluence per pulse will be increased by about three orders of magnitude. Multinucleon transfer processes look quite promising for the production and study of neutron-rich heavy nuclei located in upper part of the nuclear map not reachable by other reaction mechanisms. Reactions with actinide beams and targets are of special interest for synthesis of new neutron-enriched transfermium nuclei and not-yet-known nuclei with closed neutron shell $\mathrm{N}=126$ having the largest impact on the astrophysical r-process. The estimated cross sections for the production of these nuclei allows one to plan such experiments at currently available accelerators.
\end{abstract}

\section{Motivation}

Due to the bending of the stability line toward the neutron axis, in fusion reactions of stable nuclei one may produce only proton rich isotopes of heavy elements. For elements with $Z>100$ only neutron deficient isotopes (located to the left of the stability line) have been synthesized so far (see the left panel of Fig. 1). That is the main reason for the impossibility to reach the center of the "island of stability" $(Z \sim 110 \div 120$ and $N \sim 184)$ in fusion reactions with stable projectiles.

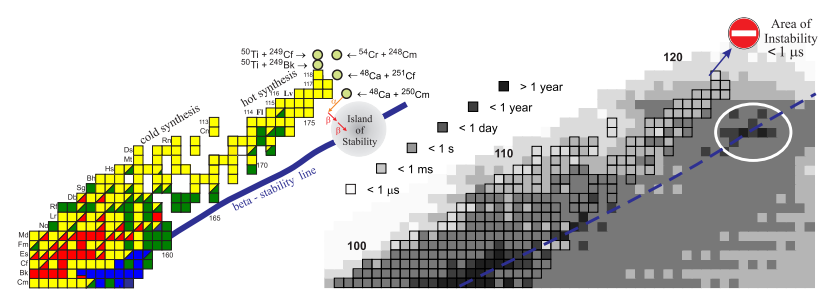

Figure 1. Upper part of the nuclear map. Current and possible experiments on synthesis of SH elements are shown. (Right panel) Predicted half-lives of SH nuclei and the "area of instability". Known nuclei are shown by the outlined rectangles.

\footnotetext{
a e-mail: zagrebaev@jinr.ru

be-mail: karpov@jinr.ru

ce-mail: greiner@fias.uni-frankfurt.de
}

Further progress in the synthesis of new elements with $Z>118$ is not quite evident. Cross sections of the "cold" fusion reactions decrease very fast with increasing charge of the projectile (they become less than $1 \mathrm{pb}$ already for $Z \geq 112[1,2])$. For the more asymmetric ${ }^{48} \mathrm{Ca}$ induced fusion reactions rather constant values (of a few picobarns) of the cross sections for the production of SH elements up to $Z=118$ were found [3]. This unusual (at first sight) behavior of the cross sections has been predicted and explained in $[4,5]$ by the relatively slow decrease of the fusion probability (in contrast to the more symmetric "cold" fusion reactions) and by the increasing survival probability of compound nuclei $(\mathrm{CN})$ owing to increasing values of their fission barriers caused by the larger shell corrections as the $\mathrm{CN}$ approach the neutron and proton closed shells in the region of the island of stability. These predictions have been fully confirmed by the experiments performed in Dubna and later in Berkeley [6] and at GSI [7, 8].

For the moment californium $(Z=98)$ is the heaviest available target that can be used in experiments. The halflife of the einsteinium isotope ${ }_{99}^{254}$ Es is 276 days, sufficient to be used as target material. However it is impossible to accumulate the required amount of this matter (several milligrams) to prepare a target. To get SH elements with $Z>118$ in a more realistic way one should proceed to heavier than ${ }^{48} \mathrm{Ca}$ projectiles. ${ }^{50} \mathrm{Ti}$ is most promising projectile for further synthesis of SH nuclei. Our calculations 
demonstrated that the use of the titanium beam instead of ${ }^{48} \mathrm{Ca}$ decreases the yield of the same SH element due to a worse fusion probability by about factor 20 [9]. Nevertheless, the elements 119 and 120 can be produced in the fusion reactions of ${ }^{50} \mathrm{Ti}$ with ${ }^{249} \mathrm{Bk}$ and ${ }^{249} \mathrm{Cf}$ targets (or in the ${ }^{54} \mathrm{Cr}+{ }^{248} \mathrm{Cm}$ fusion reaction) with the cross sections of about $0.04 \mathrm{pb}$ [9] which are already at the limit of the experimental possibilities. The first attempts to perform these experiments have been already made at GSI $[10,11]$. Only the upper limits of the cross sections have been obtained.

Synthesis of these nuclei may encounter also another important problem. The proton rich isotopes of SH elements produced in these reactions are rather short-living due to large values of $Q_{\alpha}$. Their half-lives are very close to the critical value of one microsecond needed for the $\mathrm{CN}$ to pass through the separator up to the focal plane detector. The next elements (with $Z>120$ ) being synthesized in such a way might be already beyond this natural time limit for their detection (see the right panel of Fig. 1).

\section{Fusion reactions}

Extension of the area of known isotopes of SH elements is extremely important for better understanding of their properties and for developing the models which will be able to predict well the properties of SH nuclei located beyond this area (including those at the island of stability). An important region of $\mathrm{SH}$ isotopes located between those produced in the cold and hot fusion reactions remains unstudied yet (see the gap on left panel of Fig. 1).

This gap can be filled in ordinary fusion reactions of ${ }^{48} \mathrm{Ca}$ with available lighter isotopes of $\mathrm{Pu}, \mathrm{Am}$, and $\mathrm{Cm}$. Several (rather cheap and available) isotopes of actinide elements can be used as the targets, for example, ${ }^{233,235} \mathrm{U},{ }^{239,240} \mathrm{Pu},{ }^{241} \mathrm{Am},{ }^{243} \mathrm{Cm}$, and so on [12]. It is much easier to fill the gap "from above" by the synthesis of new isotopes of $\mathrm{SH}$ elements with larger values of $\mathrm{Z}$, their subsequent $\alpha$ decay chains just fill the gap. This unexpected finding is simply explained by greater values of survival probabilities of the corresponding nuclei with $Z=114 \div 116$ as compared to those with $Z=110 \div 112$.

In spite of the decrease of the fusion probability with increasing charge number of the target nucleus, the evaporation residue (EvR) cross sections for the ${ }^{48} \mathrm{Ca}+{ }^{243} \mathrm{Cm}$ reaction was found to be higher than in reaction ${ }^{48} \mathrm{Ca}+{ }^{235} \mathrm{U}$ due to the larger survival probability of ${ }^{291} 116$ compound nucleus as compared to ${ }^{283} 112$. This means that the new isotopes of element 112 (at least, ${ }^{280,279} 112$ ) could be easier synthesized and studied as $\alpha$ decay products of the heavier elements, 114 and/or 116.

In Fig. 2 the calculated EvR cross sections are shown for the production of new isotopes of elements 115 and 116 in the ${ }^{48} \mathrm{Ca}+{ }^{241} \mathrm{Am}$ and ${ }^{48} \mathrm{Ca}+{ }^{243} \mathrm{Cm}$ fusion reactions. Note, that high intensive beam of ${ }^{40} \mathrm{Ar}$ can be obtained quite easily. This material is also much cheaper than ${ }^{48} \mathrm{Ca}$. However, as can be seen from Fig. 2, the use of an ${ }^{40} \mathrm{Ar}$ beam is less favorable as compared with ${ }^{48} \mathrm{Ca}$. This is due to much "hotter" character of the ${ }^{40} \mathrm{Ar}+{ }^{251} \mathrm{Cf}$ fusion reac- tion (only the cross sections for the 5 n evaporation channels are comparable for both reactions). The production cross sections for the proposed reactions are high enough to perform such experiments at available facilities. All the decay chains, most probably, reach finally known nuclei (see Ref. [12]). This fact significantly facilitates the identification of the new $\mathrm{SH}$ isotopes.

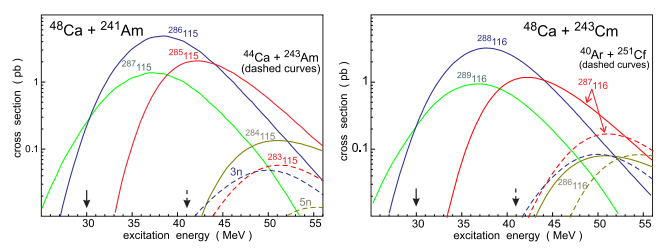

Figure 2. Production cross sections for the new isotopes of elements 115 and 116 in the ${ }^{48} \mathrm{Ca}+{ }^{241} \mathrm{Am}$ and ${ }^{48} \mathrm{Ca}+{ }^{243} \mathrm{Cm}$ fusion reactions.

Recently the synthesis of SH elements at the level of $1 \mathrm{pb}$ became more or less a routine matter for several laboratories. The corresponding experiments require about 2-week irradiation time to detect several decay chains of $\mathrm{SH}$ element. This means that many new isotopes of SH elements could be synthesized now, and the gap between nuclei produced in the cold and hot fusion reactions could be closed at last.

It is well known that there are no combinations of available projectiles and targets, the fusion of which may lead to SH nuclei located at the island of stability. Only the proton-rich isotopes of $\mathrm{SH}$ elements have been produced so far in fusion reactions (see Fig. 1). The use of radioactive ion beams cannot solve this problem because of their low intensity. Two new neutron rich isotopes of elements $116\left({ }^{294,295} 116\right)$ may be synthesized in $3 \mathrm{n}$ and $4 \mathrm{n}$ evaporation channels of the ${ }^{48} \mathrm{Ca}+{ }^{250} \mathrm{Cm}$ fusion reactions with the cross sections of about $1 \mathrm{pb}$ [12]. $\alpha$ decay chains of these nuclei lead to absolutely new neutron enriched isotopes of SH elements ended by fission of seaborgium and/or rutherfordium isotopes located already at the beta-stability line.

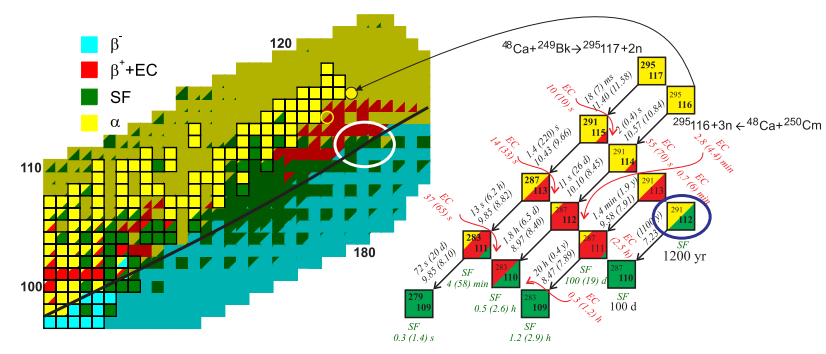

Figure 3. The pathway to the middle of the island of stability via a possible $\beta^{+}$decay of the isotopes ${ }^{291} 115$ and ${ }^{291} 114$. The first isotope may be formed after $\alpha$ decay of ${ }^{295} 117$ ( $2 \mathrm{n}$ channel of the ${ }^{48} \mathrm{Ca}+{ }^{249} \mathrm{Bk}$ fusion reaction, cross section is $0.3 \mathrm{pb}$ [9]). The second one, ${ }^{291} 114$, is formed after $\alpha$ decay of ${ }^{295} 116$ in the $3 \mathrm{n}$ evaporation channel of the ${ }^{48} \mathrm{Ca}+{ }^{250} \mathrm{Cm}$ fusion reaction with cross section of about $0.8 \mathrm{pb}$. 
Another interesting feature of the fusion reaction ${ }^{48} \mathrm{Ca}+{ }^{250} \mathrm{Cm}$ is an unexpected possibility to reach the middle of the island of stability just in ordinary fusion processes of "stable" nuclei. In this reaction new neutron enriched isotopes ${ }^{291} 114$ and ${ }^{287} 112$ are formed as $\alpha$ decay products of 3 n-evaporation residue of the corresponding $\mathrm{CN}$. These isotopes should have rather long half-lives and, thus, they could be located already in the "red" area of the nuclear map, i.e., they might be $\beta^{+}$-decaying nuclei. In Fig. 3 several possible decay chains are shown along with the corresponding values of $Q_{\alpha}$ and half-lives calculated with the use of nuclear masses predicted by A. Sobiczewski et al. [13] and by P. Möller et al. [14].

In accordance with our calculations of decay properties of SH nuclei the isotopes ${ }^{291} 114$ and ${ }^{287} 112$ may experience not only $\alpha$ decay but also electron capture with half-life of several seconds. If it is correct, the narrow pathway to the middle of the island of stability is surprisingly opened by production of these isotopes in subsequent $\alpha$-decay of elements 116 produced in the ${ }^{48} \mathrm{Ca}+{ }^{250} \mathrm{Cm} \mathrm{fu}$ sion reactions, see Fig. 3. The corresponding cross section is rather high, it is about $0.8 \mathrm{pb}$ [12]. For the moment, this is the only method which is proposed for the production of SH nuclei located just in the middle of the island of stability. Further careful study of the decay properties of unknown SH nuclei located closer to the beta-stability line is needed to confirm the existence of such a possibility.

\section{Neutron capture process}

The neutron capture process is an alternative (oldest and natural) method for the production of new heavy elements. The synthesis of heavier nuclei in the reaction of neutron capture with subsequent beta-minus decay is a well studied process. Relative yields of the isotopes formed in such a process may be found as a solution of the following set of differential equations (somewhat simplified here)

$$
\begin{aligned}
& \frac{d N_{Z, A}}{d t}=N_{Z, A-1} n_{0} \sigma_{n \gamma}^{Z, A-1}-N_{Z, A} n_{0} \sigma_{n \gamma}^{Z, A}-N_{Z, A}\left[\lambda_{Z, A}^{\beta-}\right. \\
& \left.+\lambda_{Z, A}^{f i s}+\lambda_{Z, A}^{\alpha}\right]+N_{Z-1, A} \lambda_{Z-1, A}^{\beta-}+N_{Z+2, A+4} \lambda_{Z+2, A+4}^{\alpha},
\end{aligned}
$$

where $n_{0}$ is the neutron flux (number of neutrons per square centimeter per second) and $\lambda_{Z, A}^{i}=\ln 2 / T_{1 / 2}^{i}$ is the decay rate of the nucleus $(Z, A)$ into the channel $i$ (i.e., beta-minus, alpha decays and fission).

The key quantity here is the time of neutron capture, $\tau_{n}=\left(n_{0} \sigma_{n \gamma}^{Z, A}\right)^{-1}$, where $n_{0}$ is the neutron flux (number of neutrons per square centimeter per second) and $\sigma_{n \gamma}^{Z, A}$ is the neutron capture cross section. If $\tau_{n}$ is shorter than the half-life of a given nucleus $T_{1 / 2}(Z, A)$ then the next nucleus $(Z, A+1)$ is formed by neutron capture. Otherwise the nucleus $(Z, A)$ decays before it captures next neutron. In nuclear reactors typical value of $\tau_{n} \sim 1$ year, and the nucleosynthesis occurs along the stability line by a sequence of neutron capture and $\beta^{-}$decay processes breaking at the short-living fissile fermium isotopes ${ }^{258-260} \mathrm{Fm}$ (so called "fermium gap"). In nuclear explosion $\tau_{n} \sim 1 \mu \mathrm{s}$, and more than 20 neutrons can be captured by a nucleus before it decays.

Strong neutron fluxes might be provided by nuclear reactors and nuclear explosions under laboratory conditions and by supernova explosions in nature. Theoretical models predict also another region of short-living nuclei located at $\mathrm{Z}=106 \div 108$ and $\mathrm{A} \sim 270$. In nuclear and supernova explosions (fast neutron capture) both these gaps may be bypassed if the total neutron fluence is high enough. Note that elements 99 and 100 (einsteinium and fermium) were first discovered in debris from the test thermonuclear explosion "Mike" [15].

The resulting charge number of the synthesized nuclei might be increased by sequential neutron flux exposure if two or several nuclear explosions were generated in close proximity to each other. The result depends both on the neutron fluence $n=n_{0} \tau_{\text {pulse }}$ and on the time interval between two exposures. The neutron fluence should be high enough to shift the produced neutron rich isotopes to the right from the second gap of unstable fissile nuclei located at $Z=106 \div 108$ and $A \sim 270$. Dependence on the time interval between two exposures is not so crucial if it is longer than several milliseconds (to avoid approaching the neutron drip line after several exposures) and shorter than a few minutes to avoid $\beta^{-}$decay of the produced nuclei into the area of fission instability.

Recently it was shown that the multiple rather "soft" nuclear explosions could be really used for the production of a noticeable (macroscopic) amount of neutron rich longliving SH nuclei [16]. We found a sharp increase of the probability for formation of heavy elements with $Z \geq 110$ in the multiple neutron irradiations: enhancement by several tens of orders of magnitude! The same process of multiple neutron exposures might be also realized in pulsed nuclear reactors of the next generation. An increase of the neutron fluence by about three orders of magnitude as compared with existing pulsed reactors could be quite sufficient to bypass both gaps [16].

\section{Transfer reactions}

The multinucleon transfer processes in near barrier collisions of heavy ions, in principle, allow one to produce heavy neutron rich nuclei including those located at the island of stability. These reactions were studied extensively about thirty years ago. Among other topics, there had been great interest in the use of heavy-ion transfer reactions to produce new nuclear species in the transactinide region [17-22]. The cross sections were found to decrease very rapidly with increasing atomic number of surviving heavy fragments. However, several Fm and Md isotopes have been produced at the level of $0.1 \mu \mathrm{b}$ [20].

Renewed interest in the multinucleon transfer reactions with heavy ions is caused by the limitations of other reaction mechanisms for the production of new neutron rich heavy and $\mathrm{SH}$ nuclei. Multinucleon transfer processes in near barrier collisions of heavy (and very heavy, U-like) ions seem to be the only reaction mechanism (besides the multiple neutron capture process [16]) allowing us to pro- 
duce and explore neutron rich heavy nuclei including those located at the SH island of stability.

\subsection{The Model}

Several models have been proposed and used for the description of mass transfer in deep inelastic heavy ion collisions, namely, the Focker-Planck [23] and master equations [24] for the corresponding distribution function, the Langevin equations [25], and more sophisticated semiclassical approaches [26-28]. The well known GRAZING code [29] for description of nucleon transfer reactions in heavy ion collisions is also available on the market (recently it becomes possible to run this code directly at the NRV web-site [30]). The semiclassical model used by this code describes quite well few nucleon transfer reactions. However the multinucleon transfer processes cannot be described within this model, it gives too narrow mass distributions of reaction fragments because the damped reaction channels with large kinetic energy loss are not included in this model.

Calculations performed within the microscopic timedependent Schrödinger equations [31] have clearly demonstrated that at low collision energies of heavy ions nucleons do not "suddenly jump" from one nucleus to another. Instead of that, the wave functions of valence nucleons occupy the two-center molecular states spreading gradually over volumes of both nuclei. The same adiabatic low-energy collision dynamics of heavy ions was found also within the TDHF calculations [32, 33]. This means that (1) any perturbation model based on a calculation of the sudden overlapping of single-particle wave functions of transferred nucleons (in donor and acceptor nuclei, respectively) is not applicable for description of multinucleon transfer in low-energy heavy-ion damped collisions; (2) the so called DNS model with two isolated mean fields is absolutely contrary to physics; (3) one dimensional potential energy $V(R)$ has no meaning at $R<R_{\text {contact }}$ (as well as any speculations on the depth of potential pocket of $V(R)$ ). In low-energy collisions of heavy ions at $R<R_{\text {contact }}$ the multi-dimensional potential energy (dependent on the shape parameters of the nuclear system) has to be used. The two center shell model and the adiabatic potential energy look most appropriate for the quantitative description of such processes.

The model based on the Langevin-type dynamical equations of motion was proposed recently $[34,35]$ for simultaneous description of strongly coupled multinucleon transfer, quasi-fission and fusion-fission reaction channels (difficult-to-distinguished experimentally in many cases). The distance between the nuclear centers $R$ (corresponding to the elongation of a mono-nucleus when it is formed), dynamic spheroidal-type surface deformations $\delta_{1}$ and $\delta_{2}$, the neutron and proton asymmetries, $\eta_{N}=$ $\left(2 N-N_{C N}\right) / N_{C N}, \eta_{Z}=\left(2 Z-Z_{C N}\right) / Z_{C N}$ (where $N$ and $Z$ are the neutron and proton numbers in one of the fragments, whereas $N_{C N}$ and $Z_{C N}$ refer to the whole nuclear system) are the most relevant degrees of freedom for the description of mass and charge transfer in deep inelastic scattering jointly with fusion-fission dynamics.
In low-energy damped collisions of heavy ions just the multi-dimensional potential energy surface regulates to a great extent the evolution of the nuclear system. In our approach we use a time-dependent potential energy, which after contact gradually transforms from a diabatic potential energy into an adiabatic one: $V\left(R, \delta_{1}, \delta_{2}, \eta_{N}, \eta_{Z} ; t\right)=$ $V_{\text {diab }}[1-f(t)]+V_{\text {adiab }} f(t)$ [34]. Here $t$ is the time of interaction and $f(t)$ is a smoothing function satisfying the conditions $f(t=0)=0$ and $f\left(t>>\tau_{\text {relax }}\right)=1, \tau_{\text {relax }}$ is an adjustable parameter $\sim 10^{-21} \mathrm{~s}$.

The diabatic potential energy is calculated within the double-folding procedure at the initial reaction stage, whereas in the adiabatic reaction stage we use the extended version of the two-center shell model [36], computational version of which can be found at the website [37]. Note that the diabatic, $V_{\text {diab }}$, and adiabatic, $V_{\text {adiab}}$, potential energies depend on the same variables and they are equal to each other for well separated nuclei. Thus, the total potential energy, $V\left(R, \delta_{1}, \delta_{2}, \eta_{N}, \eta_{Z} ; t\right)$, is a quite smooth function of all the parameters providing smooth driving forces, $-\partial V / \partial q_{i}$, at all reaction stages.

For the moment this approach is the only one which reproduces quite properly all the regularities of heavy ion deep inelastic scattering and quasi-fission processes $[34,35,38]$. As an example, in Fig. 4 experimental and theoretical energy-mass distributions of reaction fragments are shown formed in collisions of ${ }^{86} \mathrm{Kr}$ with ${ }^{166} \mathrm{Er}$.
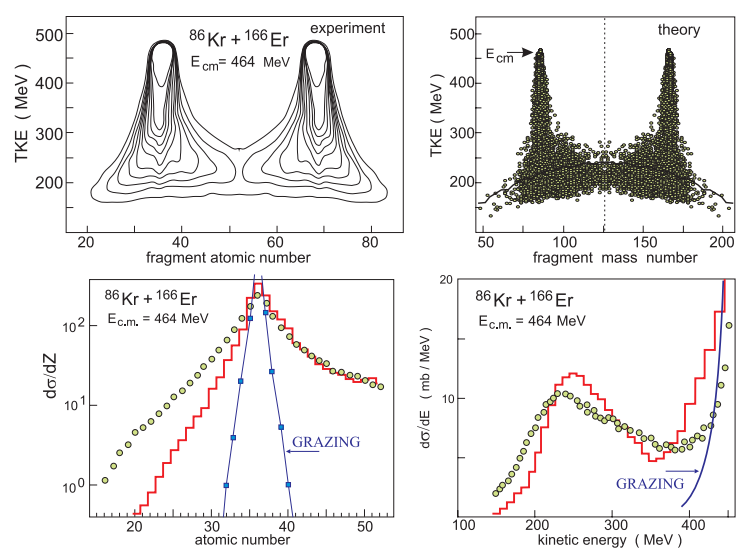

Figure 4. Charge, mass and energy distributions of reaction fragments in collisions of ${ }^{86} \mathrm{Kr}$ with ${ }^{166} \mathrm{Er}$ at $E_{\text {c.m. }}=464 \mathrm{MeV}$ [39]. The histograms indicate the calculations performed within the model described above whereas the curves show the GRAZING calculations.

\subsection{Production of transfermium nuclei}

We found that the shell effects (clearly visible in fission and quasi-fission processes) also play a noticeable role in near barrier multinucleon transfer reactions [40, 41]. These effects may significantly enhance the yield of searched-for neutron rich heavy nuclei for appropriate projectile-target combinations. In particular, the predicted process of anti-symmetrising ("inverse") quasifission may enhance the yields of long-living neutron 
rich $\mathrm{SH}$ isotopes in collisions of actinide nuclei (such as $\mathrm{U}+\mathrm{Cm}$ ). However, the role of the shell effects in damped collisions of heavy nuclei is still not absolutely clear and was not carefully studied experimentally. Very optimistic experimental results were obtained recently [42] confirming such effects in the surrogate ${ }^{160} \mathrm{Gd}+{ }^{186} \mathrm{~W}$ reaction, for which the similar "inverse quasi-fission" process $\left({ }^{160} \mathrm{Gd} \rightarrow{ }^{138} \mathrm{Ba}\right.$ while $\left.{ }^{186} \mathrm{~W} \rightarrow{ }^{208} \mathrm{~Pb}\right)$ was predicted [41].

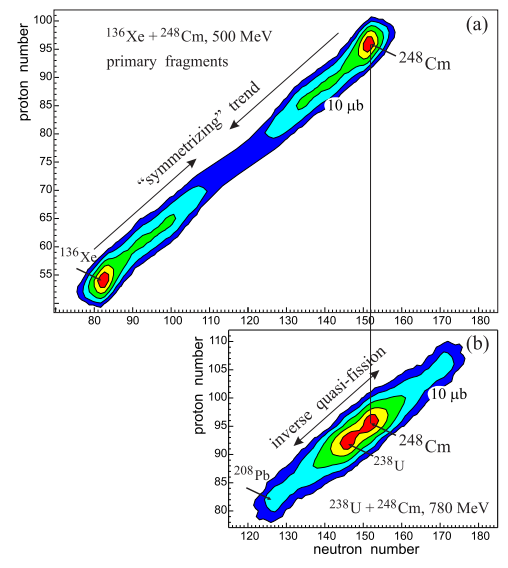

Figure 5. Calculated cross sections for the production of primary reaction fragments in collisions of ${ }^{136} \mathrm{Xe}$ (a) and ${ }^{238} \mathrm{U}$ (b) with ${ }^{248} \mathrm{Cm}$ target (contour lines are drawn over one order of magnitude).

In multinucleon transfer reactions the yields of heavier-than-target (trans-target) nuclei strongly depend on the reaction combination. Even for rather heavy projectiles (such as ${ }^{136} \mathrm{Xe}$ ) the nuclear system has a dominating symmetrizing trend of formation of reaction fragments with intermediate (heavier than projectile and lighter than target) masses (see Fig. 5).

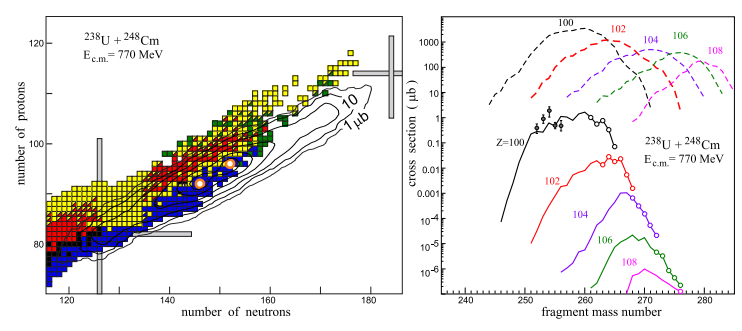

Figure 6. Cross sections for the production of primary (left panel and dashed curves) and survived (right panel) transfermium nuclei in collisions of ${ }^{238} \mathrm{U}$ with ${ }^{248} \mathrm{Cm}$ target at $E_{\text {c.m. }}=770 \mathrm{MeV}$. Open circles indicate new isotopes of transfermium elements. Experimental data are taken from [20] for the production of fermium isotopes in this reaction.

Of course, the yield of survived SH elements produced in low-energy collisions of actinide nuclei is rather low, though the shell effects give us a definite gain as compared to a monotonous exponential decrease of the cross sections with increasing number of transferred nucleons. In Fig. 6 the calculated cross sections for the production of primary and survived (evaporation residue) $\mathrm{SH}$ nuclei in damped collisions of ${ }^{238} \mathrm{U}$ with ${ }^{248} \mathrm{Cm}$ at $770 \mathrm{MeV}$ center-of-mass energy are shown along with available experimental data. As can be seen, really many new neutron-rich isotopes of SH nuclei with $Z \geq 100$ might be produced in such reactions.

The choice of collision energy is very important for the production of desired neutron-rich SH nuclei. With increasing beam energy the yield of primary fragments increases. However the excitation energy of these fragments also increases decreasing their survival probabilities. We found that the optimal beam energy for the production of neutron-rich isotopes of SH elements in multinucleon transfer reactions with heavy actinide nuclei (such as $\mathrm{U}+\mathrm{Cm}$ ) is very close to the energy needed for these nuclei to reach the contact configuration.

Actinide beams (as well as actinide targets) might be successfully used also for the production of new neutronrich heavy nuclei around the closed neutron shell $N=126$, the region having the largest impact on the astrophysical $r$ process. Near-barrier collisions of ${ }^{136} \mathrm{Xe}$ and ${ }^{192} \mathrm{Os}$ with a ${ }^{208} \mathrm{~Pb}$ target were predicted to be quite promising for the production of new neutron rich nuclei with $N \sim 126$ $[40,43]$. However low-energy collisions of stable neutron rich isotopes of elements located below lead (such as ${ }^{192} \mathrm{Os},{ }^{198} \mathrm{Pt}$ ) with available actinide targets look even more favorable.
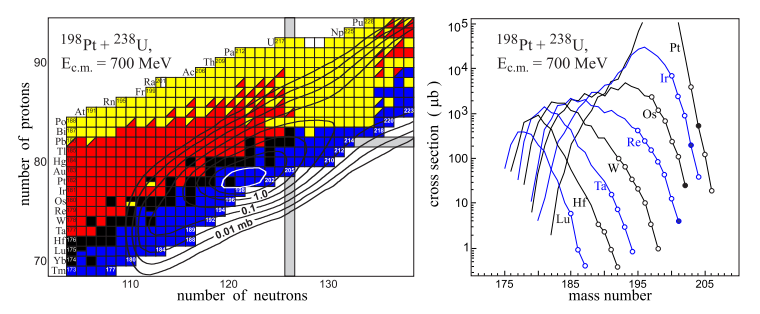

Figure 7. (Left) Contour plot of the cross sections for the formation of primary reaction fragments in collisions of ${ }^{198} \mathrm{Pt}$ with ${ }^{238} \mathrm{U}$ at $E_{\text {c.m. }}=700 \mathrm{MeV}$. (Right) Isotopic yields of elements below lead (from Lu to Pt). Circles denote not-yet-known isotopes (the solid ones show isotopes with closed neutron shell $N=126$ ).

Cross sections for the production of the isotopes of heavy elements with $Z=71 \div 78$ in low energy collisions of ${ }^{198} \mathrm{Pt}$ with ${ }^{238} \mathrm{U}$ are shown in Fig. 7. On average, the cross sections for the production of new neutron rich heavy nuclei (including those located along the closed neutron shell $N=126$ ) in this reaction are higher than in collisions of ${ }^{136} \mathrm{Xe}$ or ${ }^{192} \mathrm{Os}$ with ${ }^{208} \mathrm{~Pb}$ target.

\section{Summary}

New SH elements 119 and 120 will be synthesized very probably within a few nearest years with the use of $\mathrm{Ti}$ and/or Cr beams. Synthesis of SH elements with $\mathrm{Z}>120$ is rather problematic in near future due to extremely low cross sections and short half-lives of these elements. One might think that the epoch of ${ }^{48} \mathrm{Ca}$ in the production of 
SH nuclei was finished by the synthesis of element 118 in the ${ }^{48} \mathrm{Ca}+{ }^{249} \mathrm{Cf}$ fusion reaction [3]. However this projectile still could be successfully used for the production of new isotopes of SH elements. The extension of the area of known isotopes of SH elements is extremely important for better understanding of their properties and for developing the models which will be able to predict well the properties of SH nuclei located beyond this area. The gap of unknown SH nuclei, located between the isotopes which were produced earlier in the cold and hot fusion reactions, could be filled in fusion reactions of ${ }^{48} \mathrm{Ca}$ with available lighter isotopes of $\mathrm{Pu}, \mathrm{Am}$, and $\mathrm{Cm}$.

Then we must redirect our interests onto the production of longer living neutron enriched $\mathrm{SH}$ nuclei. New neutron-enriched isotopes of SH elements might be produced with the use of ${ }^{48} \mathrm{Ca}$ beam if a ${ }^{250} \mathrm{Cm}$ target would be prepared. In this case we get a real chance to reach the island of stability due to a possible $\beta^{+}$decay of ${ }^{291} 114$ and ${ }^{287} 112$ nuclei formed in this reaction. The same path to the island of stability is opened also in the $2 \mathrm{n}$ evaporation channel of the ${ }^{48} \mathrm{Ca}+{ }^{249} \mathrm{Bk}$ fusion reaction leading to the isotope ${ }^{291} 115$ having a chance for $\beta^{+}$decay.

Low-energy multinucleon transfer reactions look quite appropriate for the production of new neutron-enriched heavy nuclei. Reactions with actinide beams and targets are of special interest for synthesis of new neutronenriched transfermium nuclei and not-yet-known nuclei with the closed neutron shell $N=126$ having the largest impact on the astrophysical r-process. However, it is rather difficult to perform these experiments because of the low beam intensities of the massive projectiles and problems with separation and detection of heavy reaction products. The available experimental data on the production of heavy nuclei in low-energy multinucleon transfer reactions are still insufficient and fragmentary to make accurate predictions. Urgently needed are new experiments, including those in which the role of shell effects in reaction dynamics can be clarified much better.

\section{References}

[1] S. Hofmann and G. Münzenberg, Rev. Mod. Phys. 72, 733 (2000).

[2] K. Morita et al., J. Phys. Soc. Jpn. 76, 043201 (2007).

[3] Yu.Ts. Oganessian, V.K. Utyonkov et al., Phys. Rev. C 74, 044602 (2006).

[4] V.I. Zagrebaev et al., Phys. At. Nucl. 66, 1033 (2003)

[5] V.I. Zagrebaev, Nucl. Phys. A 734, 164 (2004).

[6] P.A. Ellison, et al., Phys. Rev. Lett. 105, 182701 (2010).

[7] J.M. Gates et al., Phys. Rev. C 83, 054618 (2011).

[8] S. Hofmann et al., Eur. Phys. J. A 48, 62 (2012).
[9] V.I. Zagrebaev and W. Greiner, Phys. Rev. C 78, 034610 (2008).

[10] S. Hofmann et al., GSI Report 2012-1, 202 (2012).

[11] J. Khuyagbaatar et al., GSI Report 2012-1, 131 (2013).

[12] V.I. Zagrebaev, A.V. Karpov and W. Greiner, Phys. Rev. C 85, 014608 (2012).

[13] I. Muntian, Z. Patyk and A. Sobiczewski, Phys. At. Nucl. 66, 1015 (2003).

[14] P. Möller, J.R. Nix, K.-L. Kratz, At. Data Nucl. Data Tables 66, 131 (1997).

[15] H. Diamond et al., Phys. Rev. 119, 2000 (1960).

[16] V.I. Zagrebaev, A.V. Karpov, I.N. Mishustin and W. Greiner, Phys. Rev. C 84, 044617 (2011).

[17] E.K. Hulet, et al., Phys. Rev. Lett. 39, 385 (1977).

[18] H. Essel et al., Z. Phys. A 289, 265 (1979).

[19] H. Freiesleben et al., Z. Phys. A 292, 171 (1979).

[20] M. Schädel et al., Phys. Rev. Lett. 48, 852 (1982).

[21] K.J. Moody et al., Phys. Rev. C 33, 1315 (1986).

[22] R.B. Welch et al., Phys. Rev. C 35, 204 (1987).

[23] W. Nörenberg, Phys. Lett. B 52, 289 (1974).

[24] L.G. Moretto and J.S. Sventek, Phys. Lett. B 58, 26 (1975).

[25] P. Fröbrich and S.Y. Xu, Nucl. Phys. A 477, 143 (1988).

[26] E. Vigezzi and A. Winther, Ann. Phys. (N.Y.) 192, 432 (1989).

[27] V.I. Zagrebaev, Ann. Phys. (N.Y.) 197, 33 (1990).

[28] A. Winther, Nucl. Phys. A 594, 203 (1995).

[29] http://personalpages.to.infn.it/ nanni/grazing/.

[30] http://nrv.jinr.ru/nrv/webnrv/grazing/.

[31] V.I. Zagrebaev, V.V. Samarin and W. Greiner, Phys. Rev. C 75, 035809 (2007).

[32] C. Simenel, A. Wakhle and B. Avez, J. Phys.: Conf. Ser. 20, 012118 (2013).

[33] K. Sekizawa and K. Yabana, Phys. Rev. C 88, 014614 (2013).

[34] V. Zagrebaev, W. Greiner, J. Phys. G 31, 825 (2005).

[35] V. Zagrebaev, W. Greiner, J. Phys. G 34, 1 (2007).

[36] V. Zagrebaev, et al., Phys. Part. Nucl. 38, 469 (2007).

[37] V. Zagrebaev, A. Alekseev and A. Karpov, Two Center Shell Model code of NRV, http://nrv.jinr.ru/nrv/.

[38] Y. Aritomo, Phys. Rev. C 80, 064604 (2009).

[39] A. Gobbi et al., in Proc. Int. School of Phys. "Enrico Fermi", Varenna, 1979 (North-Holland, 1981) 1.

[40] V. Zagrebaev and W. Greiner, Phys. Rev. Lett. 101, 122701 (2008).

[41] V. Zagrebaev and W. Greiner, J. Phys. G 34, 2265 (2007).

[42] W. Loveland et al., Phys. Rev. C 83, 044610 (2011).

[43] V.I. Zagrebaev and W. Greiner, Phys. Rev. C 83, 044618 (2011). 